\title{
Distribuição do sistema radicular de cafeeiro conilon irrigado e não irrigado
}

\author{
André Monzoli Covre(1), Fábio Luiz Partelli(1), Ivoney Gontijo(1) e Moises Zucoloto(1) \\ (1)Universidade Federal do Espírito Santo, Centro Universitário Norte do Espírito Santo, Departamento de Ciências Agrárias \\ e Biológicas, Rodovia BR 101 Norte, Km 60, Bairro Litorâneo, CEP 29932-540 São Mateus, ES, Brasil. E-mail: andre-covre@hotmail.com, \\ partelli@yahoo.com.br, ivoney.gontijo@ufes.br, moises.zucoloto@ufes.br
}

\begin{abstract}
Resumo - O objetivo deste trabalho foi avaliar a distribuição do sistema radicular do cafeeiro conilon (Coffea canephora) irrigado e não irrigado. Utilizaram-se plantas de conilon da variedade clonal Emcapa 8111, genótipo 02, com cinco anos de idade. Utilizou-se o delineamento inteiramente casualizado, em parcela subsubdividida, com cinco repetições. Os tratamentos consistiram de: presença e ausência de irrigação (parcelas), 13 distâncias do tronco (dez no sentido da entrelinha e três no sentido da linha de plantio) e seis camadas de solo $(0-60 \mathrm{~cm})$ nas subparcelas. As raízes foram coletadas, lavadas, digitalizadas e processadas pelo programa Safira, para quantificar área superficial, comprimento, volume e diâmetro. As plantas de conilon não irrigadas apresentaram maiores área superficial, comprimento e volume de raízes por volume de solo, bem como distribuição de raízes menos discrepante na superfície do solo e em profundidade, em comparação às plantas irrigadas. O sistema de irrigação por gotejamento promove maior distribuição de raízes nas plantas irrigadas, na zona compreendida pelo bulbo úmido da irrigação.
\end{abstract}

Termos para indexação: Coffea canephora, deficit hídrico, irrigação por gotejamento, programa Safira.

\section{Root system distribution of irrigated and nonirrigated conilon coffee}

\begin{abstract}
The objective of this work was to evaluate the distribution of the root system of irrigated and nonirrigated conilon coffee (Coffea canephora). Conilon plants of the clonal variety Emcapa 8111, genotype 02, with five years of age, were used. The design was completely randomized in split-split plots with five replicates. The treatments consisted of: presence and absence of irrigation (plots), 13 distances from the stem (ten in the direction between rows and three of the planting row) and six soil layers $(0-60 \mathrm{~cm})$ in the split-plots. Roots were collected, washed, scanned, and processed by the Safira software to quantify surface area, length, volume, and diameter. Nonirrigated conilon plants showed greater root surface area, length, and volume per soil volume, as well as less discrepant distribution of roots in the soil surface and in depth, in comparison to irrigated plants. The drip irrigation system promotes higher root distribution in irrigated plants, in the area covered by the wet-bulb irrigation.
\end{abstract}

Index terms: Coffea canephora, water deficit, trickle irrigation, Safira software.

\section{Introdução}

O cultivo do cafeeiro conilon (Coffea canephora Pierre ex A. Froehner) tem-se expandido para áreas onde a deficiência hídrica é o principal fator limitante ao crescimento e à produção (Silva et al., 2010; Araujo et al., 2011; Partelli et al., 2013), o que torna necessário realizá-lo predominantemente sob irrigação. Segundo DaMatta \& Ramalho (2006), em diversos países produtores de café, o deficit hídrico é considerado o principal estresse ambiental capaz de afetar o desenvolvimento e a produção do cafeeiro. Os principais mecanismos fisiológicos de tolerância ao deficit hídrico em genótipos de $C$. canephora são a eficiência na retirada de água do solo, o controle estomático e a redução da área foliar para manutenção da transpiração (Pinheiro et al., 2004; Marraccini et al., 2012; Silva et al., 2013). Genótipos com produção relativamente elevada, submetidos ao deficit hídrico, são capazes de manter potenciais hídricos foliares adequados, por meio da combinação entre o aprofundamento do sistema radicular e o aumento do controle estomático (Silva et al., 2010).

O sistema radicular do cafeeiro varia de acordo com espécie, genótipo, idade da planta, estação do ano, clima, densidade da cultura, estresses bióticos, textura e estrutura do solo (Partelli et al., 2014; Ronchi et al., 2015). Conforme Rena \& Guimarães (2000), o sistema 
radicular do cafeeiro é pseudopivotante, pois, na maioria dos casos, apresenta raízes pivotantes curtas, grossas e que terminam abruptamente, e raramente estende-se mais que $45 \mathrm{~cm}$ abaixo da superfície do solo. Segundo Barreto et al. (2006), o aprofundamento radicular é uma característica fenotípica importante para o cafeeiro e pode proporcionar maior resistência ao deficit hídrico. É importante salientar que a irrigação afeta o padrão de crescimento radicular do cafeeiro, com redução na profundidade de penetração das raízes, o que estimula o desenvolvimento de raízes primárias e secundárias nas camadas mais superficiais do solo (Belan et al., 2011). Ronchi et al. (2015) afirmam que a maior parte do sistema radicular está distribuída na camada superficial do solo e Silva et al. (2009) relatam que as raízes existentes nessa camada são as mais importantes na extração de água em plantas de café.

Diversas tecnologias têm contribuído para a obtenção de produtividades superiores a $9,0 \mathrm{mil} \mathrm{kg} \mathrm{ha}^{-1}$ de café beneficiado em lavouras de cafeeiro conilon, com destaque para o uso correto de calagem, fertilizantes, sistema de poda, adensamento, controle fitossanitário e irrigação. Dessa forma, o conhecimento da distribuição do sistema radicular do cafeeiro é de grande importância para o manejo da lavoura, já que raízes bem desenvolvidas podem promover melhor absorção e aproveitamento de água, nutrientes, fungicidas e inseticidas, o que pode influenciar diretamente a produtividade da planta e a tolerância ao deficit hídrico (Carvalho et al., 2008; Partelli et al., 2014). Além disso, esse conhecimento pode ser usado para estimar a necessidade de água do cafeeiro (Silva et al., 2009) e para definir o melhor sistema de irrigação (Barreto et al., 2006).

O objetivo deste trabalho foi avaliar a distribuição do sistema radicular do cafeeiro conilon (Coffea canephora) irrigado e não irrigado.

\section{Material e Métodos}

O experimento foi conduzido no município de Itabela, no Sul do estado da Bahia $\left(16^{\circ} 42^{\prime} 13^{\prime \prime S}\right.$, $39^{\circ} 25^{\prime} 28^{\prime \prime} \mathrm{W}$, a $108 \mathrm{~m}$ de altitude). De acordo com a classificação de Köppen, o clima é do tipo Aw, tropical, com estação seca no inverno e chuvosa no verão. O solo é classificado como Latossolo Amarelo originalmente distrófico (Santos et al., 2013), cujas características químicas e físicas estão descritas na Tabela 1.
As mudas de cafeeiro conilon foram obtidas a partir de estacas de brotos ortotrópicos do genótipo 02 da variedade clonal Emcapa 8111 (Bragança et al., 2001) e foram plantadas quando apresentavam cinco pares de folhas, em março de 2008, no espaçamento de 3,5x1,0 $\mathrm{m}$. Durante a implantação da lavoura, foram aplicados $10 \mathrm{~L}$ de palha de café, $5,0 \mathrm{~L}$ de esterco bovino e $50 \mathrm{~g}$ de $\mathrm{P}_{2} \mathrm{O}_{5}$ por metro de sulco, além de $1,0 \mathrm{Mg} \mathrm{ha}^{-1}$ de calcário dolomítico espalhado no sulco de plantio.

Utilizou-se o delineamento inteiramente casualizado, em arranjo de parcela subsubdividida, com cinco repetições. Os tratamentos consistiram de: irrigação e não irrigação dos cafeeiros nas parcelas, 13 distâncias de coleta do tronco do cafeeiro $(17,33,50,67,83$, $100,117,133,150$ e $167 \mathrm{~cm}$ no sentido da entrelinha e 17,33 e $50 \mathrm{~cm}$ no sentido da linha de plantio) e seis camadas de solo $(0-10,10-20,20-30,30-40,40-50$ e 50-60 cm) nas subparcelas. Para a avaliação do sistema radicular aos 66 meses após o plantio, foram selecionadas plantas com diâmetro de copa igual ou superior a 2,5 m, vigorosas e sem sintomas de doenças e deficiência nutricional. Portanto, as raízes foram coletadas no final de setembro de 2013, período de grande crescimento vegetativo do cafeeiro na região.

O sistema de irrigação utilizado foi o de gotejamento superficial, com uma linha de emissores espaçados em $0,5 \mathrm{~m}$ e vazão de $2,0 \mathrm{~L} \mathrm{~h}^{-1}$. A linha de emissores localizava-se a $5,0 \mathrm{~cm}$ do tronco do cafeeiro, no sentido da entrelinha de plantio. No tratamento irrigado, o manejo de irrigação adotado foi por balanço hídrico diário, com base na evapotranspiração da cultura, na precipitação pluvial medida no local e nas características de armazenamento de água do solo. Já no tratamento não irrigado, a água de irrigação foi suspensa quando as plantas tinham 36 meses, com cultivo em condições de sequeiro por 30 meses (entre março de 2011 e outubro de 2013), para melhor aclimatação às condições de deficit hídrico.

Os tratos culturais da lavoura consistiram basicamente em controle de plantas daninhas com herbicidas e roçadeira, manejo fitossanitário preventivo, calagem, adubação e irrigação (somente na parcela irrigada). As adubações de produção foram realizadas com a aplicação de $500 \mathrm{~kg} \mathrm{ha}^{-1}$ por ano de $\mathrm{N}, 100 \mathrm{~kg} \mathrm{ha}^{-1}$ por ano de $\mathrm{P}_{2} \mathrm{O}_{5}, 400 \mathrm{~kg} \mathrm{ha}^{-1}$ por ano de $\mathrm{K}_{2} \mathrm{O}$ e $1,0 \mathrm{Mg} \mathrm{ha}^{-1}$ por ano de calcário dolomítico. Nas plantas irrigadas, a aplicação destes nutrientes foi realizada semanalmente, por meio de 
fertirrigação. Nas plantas não irrigadas, as adubações foram realizadas a lanço, na projeção da copa a aproximadamente $1,0 \mathrm{~m}$ do tronco, e parceladas em cinco aplicações por ano, em setembro, novembro, janeiro, março e junho. A lavoura foi conduzida no sistema de poda programada de ciclo, com quatro hastes ortotrópicas conforme Verdin Filho et al. (2014); contudo, a lavoura foi avaliada antes de atingir a fase de poda.

Após a definição do local, monólitos de solo com raízes com volume de $27 \mathrm{~cm}^{3}$ foram coletados em cada camada de solo, com trado tipo sonda. As amostras coletadas foram armazenadas cuidadosamente em sacos de plástico e mantidas em câmara fria, a aproximadamente $-10^{\circ} \mathrm{C}$, até a lavagem, sob água corrente, em peneira de 30 mesh, para separação das raízes. As raízes foram digitalizadas com câmera digital Sony de 18.2 Megapixels e, posteriormente, analisadas pelo programa Safira (Embrapa Instrumentação Agropecuária, São Carlos, SP). Assim, quantificou-se a área superficial $\left(\mathrm{mm}^{2} \mathrm{~cm}^{-3}\right)$, o comprimento $\left(\mathrm{mm} \mathrm{cm}^{-3}\right)$, o volume

Tabela 1. Características químicas e granulométricas de seis profundidades do solo (Latossolo Amarelo) em área cultivada com cafeeiro conilon (Coffea canephora) irrigado e não irrigado, no Estado da Bahia.

\begin{tabular}{|c|c|c|c|c|c|c|}
\hline \multirow{2}{*}{ Atributos químicos } & \multicolumn{6}{|c|}{ Camada de solo $(\mathrm{cm})$} \\
\hline & $0-10$ & $10-20$ & $20-30$ & $30-40$ & $40-50$ & $50-60$ \\
\hline & \multicolumn{6}{|c|}{ Irrigado } \\
\hline $\mathrm{P}\left(\mathrm{mg} \mathrm{dm}^{-3}\right)$ & 19 & 6 & 3 & 1 & 1 & 1 \\
\hline $\mathrm{K}\left(\mathrm{mg} \mathrm{dm} \mathrm{m}^{-3}\right)$ & 49 & 55 & 47 & 45 & 37 & 32 \\
\hline $\mathrm{S}\left(\mathrm{mg} \mathrm{dm}^{-3}\right)$ & 5 & 6 & 9 & 17 & 23 & 36 \\
\hline $\mathrm{Ca}\left(\mathrm{cmol}_{\mathrm{c}} \mathrm{dm}^{-3}\right)$ & 2,6 & 1,8 & 1,5 & 0,7 & 0,5 & 0,4 \\
\hline $\mathrm{Mg}\left(\mathrm{cmol}_{\mathrm{c}} \mathrm{dm}^{-3}\right)$ & 1,0 & 0,8 & 0,6 & 0,3 & 0,2 & 0,1 \\
\hline $\mathrm{Al}\left(\mathrm{cmol}_{\mathrm{c}} \mathrm{dm}^{-3}\right)$ & 0,0 & 0,0 & 0,0 & 0,4 & 0,7 & 0,8 \\
\hline $\mathrm{H}+\mathrm{Al}\left(\mathrm{cmol}_{\mathrm{c}} \mathrm{dm}^{-3}\right)$ & 1,3 & 1,2 & 1,6 & 2,9 & 3,6 & 4,7 \\
\hline $\mathrm{pH}$ & 6,8 & 6,1 & 6,1 & 5,1 & 4,8 & 4,7 \\
\hline Matéria orgânica (dag kg-1) & 2,4 & 1,7 & 1,2 & 0,9 & 0,7 & 0,5 \\
\hline $\mathrm{Fe}\left(\mathrm{mg} \mathrm{dm}^{-3}\right)$ & 279 & 340 & 341 & 417 & 347 & 274 \\
\hline $\mathrm{Zn}\left(\mathrm{mg} \mathrm{dm} \mathrm{m}^{-3}\right)$ & 2,7 & 1,0 & 0,5 & 0,3 & 0,2 & 0,2 \\
\hline $\mathrm{Cu}\left(\mathrm{mg} \mathrm{dm}^{-3}\right)$ & 1,3 & 0,7 & 0,4 & 0,3 & 0,2 & 0,2 \\
\hline $\operatorname{Mn}\left(\mathrm{mg} \mathrm{dm}^{-3}\right)$ & 19 & 4 & 2 & 2 & 2 & 1 \\
\hline $\mathrm{B}\left(\mathrm{mg} \mathrm{dm} \mathrm{m}^{-3}\right)$ & 0,61 & 0,71 & 0,65 & 0,61 & 0,49 & 0,43 \\
\hline \multirow[t]{2}{*}{$\mathrm{Na}\left(\mathrm{mg} \mathrm{dm}^{-3}\right)$} & 25 & 24 & 23 & 22 & 18 & 15 \\
\hline & \multicolumn{6}{|c|}{ Não irrigado } \\
\hline $\mathrm{P}\left(\mathrm{mg} \mathrm{dm}^{-3}\right)$ & 18 & 14 & 12 & 8 & 5 & 2 \\
\hline $\mathrm{K}\left(\mathrm{mg} \mathrm{dm} \mathrm{m}^{-3}\right)$ & 50 & 56 & 52 & 49 & 46 & 44 \\
\hline $\mathrm{S}\left(\mathrm{mg} \mathrm{dm}^{-3}\right)$ & 11 & 9 & 6 & 13 & 30 & 14 \\
\hline $\mathrm{Ca}\left(\mathrm{cmol}_{\mathrm{c}} \mathrm{dm}^{-3}\right)$ & 2,1 & 1,8 & 1,3 & 1,0 & 0,9 & 0,8 \\
\hline $\mathrm{Mg}\left(\mathrm{cmol}_{\mathrm{c}} \mathrm{dm}^{-3}\right)$ & 0,7 & 0,6 & 0,5 & 0,4 & 0,3 & 0,3 \\
\hline $\mathrm{Al}\left(\mathrm{cmol}_{\mathrm{c}} \mathrm{dm}^{-3}\right)$ & 0,0 & 0,0 & 0,0 & 0,3 & 0,3 & 0,4 \\
\hline $\mathrm{H}+\mathrm{Al}\left(\mathrm{cmol}_{\mathrm{c}} \mathrm{dm}^{-3}\right)$ & 1,6 & 1,6 & 1,7 & 2,2 & 2,2 & 2,6 \\
\hline $\mathrm{pH}$ & 6,0 & 6,1 & 5,8 & 5,3 & 5,3 & 5,1 \\
\hline Matéria orgânica (dag kg-1) & 3,1 & 1,9 & 1,6 & 1,2 & 0,9 & 0,7 \\
\hline $\mathrm{Fe}\left(\mathrm{mg} \mathrm{dm}^{-3}\right)$ & 107 & 174 & 301 & 341 & 398 & 362 \\
\hline $\mathrm{Zn}\left(\mathrm{mg} \mathrm{dm} \mathrm{m}^{-3}\right)$ & 2,7 & 2,0 & 1,6 & 1,1 & 0,8 & 0,5 \\
\hline $\mathrm{Cu}\left(\mathrm{mg} \mathrm{dm}^{-3}\right)$ & 1,0 & 0,8 & 0,7 & 0,6 & 0,5 & 0,1 \\
\hline $\operatorname{Mn}\left(\mathrm{mg} \mathrm{dm}^{-3}\right)$ & 10 & 7 & 3 & 2 & 2 & 1 \\
\hline $\mathrm{B}\left(\mathrm{mg} \mathrm{dm}^{-3}\right)$ & 0,73 & 0,81 & 0,87 & 0,56 & 0,53 & 0,49 \\
\hline $\mathrm{Na}\left(\mathrm{mg} \mathrm{dm}^{-3}\right)$ & 29 & 27 & 24 & 22 & 20 & 19 \\
\hline \multicolumn{7}{|l|}{ Frações granulométricas } \\
\hline Areia total $\left(\mathrm{g} \mathrm{kg}^{-1}\right)$ & 732 & 728 & 632 & 528 & 510 & 478 \\
\hline Silte $\left(\mathrm{g} \mathrm{kg}^{-1}\right)$ & 108 & 112 & 128 & 132 & 150 & 102 \\
\hline $\operatorname{Argila}\left(\mathrm{g} \mathrm{kg}^{-1}\right)$ & 160 & 160 & 240 & 340 & 340 & 420 \\
\hline
\end{tabular}


$\left(\mathrm{mm}^{3} \mathrm{~cm}^{-3}\right)$, por volume de solo, e o diâmetro das raízes $(\mathrm{mm})$.

Os dados de área superficial, comprimento, volume e diâmetro das raízes, por não apresentarem distribuição normal, foram transformados pela função $\mathrm{y}=\log (\mathrm{x}+11)$, antes da análise de variância e da comparação de médias. Entretanto, os dados foram apresentados na forma original. Os dados obtidos após a transformação Box-Cox foram submetidos à análise de variância, e as médias foram comparadas pelo teste de Tukey, a 5\% de probabilidade, com o auxílio do programa Assistat, versão 7.7 beta (Universidade Federal de Campina Grande, Campina Grande, PB). Para visualizar a distribuição espacial do sistema radicular no perfil do solo em duas dimensões e nas diferentes distâncias e profundidades, foram confeccionados gráficos de isolinhas com o programa GS+, versão 7.0 (Gamma Design Software, Plainwell, MI, EUA).

\section{Resultados e Discussão}

Houve interação significativa entre as características área superficial, comprimento, volume e diâmetro das raízes por volume de solo para os tratamentos: irrigação e não irrigação e distâncias do tronco do cafeeiro (Tabela 2); irrigação e não irrigação e camadas de solo (Tabela 3); e distâncias do tronco do cafeeiro e camadas de solo (Tabela 4). No entanto, não houve interação entre os tratamentos irrigação e não irrigação e distâncias do tronco do cafeeiro e camadas de solo, simultaneamente.

As plantas de cafeeiro conilon não irrigadas apresentaram área superficial, comprimento e volume de raízes superiores aos das plantas irrigadas, principalmente nas distâncias de 17 e $33 \mathrm{~cm}$ do tronco do cafeeiro, no sentido da linha de plantio, e na de $50 \mathrm{~cm}$ do tronco do cafeeiro, no sentido da entrelinha (Tabela 2). Quanto às camadas de solo, observou-se que as plantas não irrigadas apresentaram maiores área superficial e comprimento de raízes na camada de $0-10 \mathrm{~cm}$, além de maior volume de raízes nas camadas de $0-10$ e $10-20 \mathrm{~cm}$ de profundidade, em comparação às plantas irrigadas (Tabela 3 ).

Verificaram-se maiores área superficial, comprimento, volume e diâmetro de raízes nas distâncias horizontais mais próximas do tronco do cafeeiro, tanto no sentido da linha de plantio quanto da entrelinha, e nas primeiras camadas do solo (Tabela 4). Houve redução na área superficial, no comprimento e no volume de raízes com o aumento da distância do tronco do cafeeiro, principalmente na região da entrelinha de plantio do cafeeiro e com o aumento

Tabela 2. Desdobramento da interação significativa entre os tratamentos irrigado e não irrigado e distâncias do tronco do cafeeiro, quanto às variáveis área superficial, comprimento e volume de raízes, em plantas de cafeeiro conilon (Coffea canephora) $)^{(1)}$.

\begin{tabular}{|c|c|c|c|c|c|c|}
\hline \multirow{2}{*}{$\begin{array}{l}\text { Distância } \\
(\mathrm{cm})\end{array}$} & \multicolumn{2}{|c|}{ Área superficial $\left(\mathrm{mm}^{2} \mathrm{~cm}^{-3}\right)$} & \multicolumn{2}{|c|}{ Comprimento $\left(\mathrm{mm} \mathrm{cm}^{-3}\right)$} & \multicolumn{2}{|c|}{ Volume $\left(\mathrm{mm}^{3} \mathrm{~cm}^{-3}\right)$} \\
\hline & Irrigado & Não irrigado & Irrigado & Não irrigado & Irrigado & Não irrigado \\
\hline & \multicolumn{6}{|c|}{ Sentido da linha de plantio } \\
\hline 17 & $404 \mathrm{Bab}$ & $962 \mathrm{Aab}$ & 137Bab & $283 \mathrm{Aab}$ & 117Babc & $329 \mathrm{Aab}$ \\
\hline 33 & $474 \mathrm{Bab}$ & $1315 \mathrm{Aa}$ & $147 \mathrm{Bab}$ & 407Aa & $153 \mathrm{Babc}$ & $402 \mathrm{Aa}$ \\
\hline \multirow[t]{2}{*}{50} & $542 \mathrm{Aab}$ & $802 \mathrm{Abc}$ & $165 \mathrm{Aab}$ & $250 \mathrm{Ab}$ & $180 \mathrm{Aab}$ & $241 \mathrm{Abc}$ \\
\hline & \multicolumn{6}{|c|}{ Sentido da entrelinha de plantio } \\
\hline 17 & 759Aa & $828 \mathrm{Abc}$ & $233 \mathrm{Aa}$ & $287 \mathrm{Aab}$ & 244Aa & 207Abcd \\
\hline 33 & $358 \mathrm{Aab}$ & 458Acd & 114Aab & $152 \mathrm{Abc}$ & 104Aabc & 123Acde \\
\hline 50 & $192 \mathrm{Bb}$ & 517Abcd & $66 \mathrm{Bb}$ & $177 \mathrm{Abc}$ & 49Bbc & 143Acde \\
\hline 67 & $207 \mathrm{Ab}$ & 473Acd & $69 \mathrm{Ab}$ & $151 \mathrm{Abc}$ & $56 \mathrm{Abc}$ & 140Acde \\
\hline 83 & $191 \mathrm{Ab}$ & $252 \mathrm{Ad}$ & $63 \mathrm{Ab}$ & $84 \mathrm{Ac}$ & $52 \mathrm{Abc}$ & 67Ade \\
\hline 100 & $158 \mathrm{Ab}$ & $251 \mathrm{Ad}$ & $52 \mathrm{Ab}$ & $85 \mathrm{Ac}$ & $44 \mathrm{Abc}$ & 65Ade \\
\hline 117 & $188 \mathrm{Ab}$ & 197Ad & $61 \mathrm{Ab}$ & $84 \mathrm{Ac}$ & $55 \mathrm{Abc}$ & $54 \mathrm{Ae}$ \\
\hline 133 & $93 \mathrm{Ab}$ & $202 \mathrm{Ad}$ & $28 \mathrm{Ab}$ & $66 \mathrm{Ac}$ & $33 \mathrm{Ac}$ & $58 \mathrm{Ae}$ \\
\hline 150 & $125 \mathrm{Ab}$ & $163 \mathrm{Ad}$ & $43 \mathrm{Ab}$ & 49Ac & $33 \mathrm{Ac}$ & 46Ae \\
\hline 167 & $130 \mathrm{Ab}$ & 280Ad & $43 \mathrm{Ab}$ & $94 \mathrm{Ac}$ & $38 \mathrm{Abc}$ & 75Ade \\
\hline CV geral (\%) & \multicolumn{2}{|c|}{49,1} & \multicolumn{2}{|c|}{44,2} & \multicolumn{2}{|c|}{45,4} \\
\hline
\end{tabular}

${ }^{(1)}$ Médias seguidas de letras iguais, maiúsculas nas linhas e minúsculas nas colunas, não diferem pelo teste de Tukey, a 5\% de probabilidade. 
da profundidade das camadas de solo (Tabelas 2, 3 e 4). Essas tendências também foram observadas na distribuição espacial das raízes nas camadas de solo (Figuras 1, 2 e 3). Resultados semelhantes foram obtidos por Ronchi et al. (2015), que constataram maiores área superficial, comprimento, volume e matéria seca de raízes em cafeeiro arábica, em franco crescimento, nas posições mais próximas do tronco da planta, o que está de acordo com os resultados do presente trabalho. Contudo, Partelli et al. (2014) relataram maiores área superficial, comprimento e volume de raízes na região da entrelinha de plantio, em C. Canephora, aos 146 meses de idade, cultivado em sistema adensado.

Maiores área superficial, comprimento e volume de raízes das plantas irrigadas foram observados na região do bulbo de irrigação (bulbo úmido), que compreende toda a região do sentido da linha e os primeiros centímetros da entrelinha de plantio (Tabela 2 e Figuras 1,2 e 3). Como as plantas irrigadas foram adubadas por meio da fertirrigação, pode-se inferir que as raízes tendem a se concentrar na região mais próxima à linha de emissores. Sakai et al. (2015) também encontraram maior massa de matéria seca de raízes (em $\mathrm{g} \mathrm{dm}^{-3}$ ) na região próxima aos emissores, em cafeeiro arábica irrigado, quando comparado ao sistema de sequeiro. Ronchi et al. (2015) atribuíram maiores área superficial, comprimento, volume e massa de matéria seca de raízes de cafeeiro arábica fertirrigado, nessa mesma camada, à distribuição de fertilizantes de modo concentrado, via fertirrigação.

Maiores área superficial, comprimento e volume de raízes em plantas irrigadas podem ter ocorrido como resposta fisiológica do sistema radicular, que apresentou maior desenvolvimento em locais de melhor fertilidade e disponibilidade hídrica (Lynch, 1995; Partelli et al., 2014). De acordo com Sakai et al. (2015), a disponibilidade de água e nutrientes na região do bulbo úmido aumenta o desenvolvimento de raízes e a formação de pelos radiculares, o que foi confirmado pela distribuição espacial das raízes no perfil do solo avaliado (Figuras 1, 2 e 3). Portanto, considera-se que a profundidade efetiva do sistema radicular se altera com a adoção da fertirrigação, bem como com o posicionamento e o espaçamento entre os emissores (Barreto et al., 2006).

As plantas não irrigadas apresentaram distribuição de raízes menos discrepante em relação às plantas irrigadas e maior aprofundamento do sistema radicular no perfil do solo, especialmente no sentido da linha de plantio (Figuras 1, 2 e 3). Conforme Santos \& Carlesso (1998), o deficit hídrico estimula a expansão do sistema radicular para zonas mais profundas e úmidas do solo, em razão da maior evapotranspiração na camada superficial do solo (Hoogenboom et al., 1987). Já Sakai et al. (2015) constataram maior enraizamento no

Tabela 3. Desdobramento da interação significativa entre os tratamentos irrigado e não irrigado e camadas de solo, quanto as variáveis área superficial, comprimento e volume de raízes, em plantas de cafeeiro conilon (Coffea canephora) ${ }^{(1)}$.

\begin{tabular}{|c|c|c|c|c|c|c|}
\hline \multirow{2}{*}{$\begin{array}{l}\text { Camada de solo } \\
(\mathrm{cm})\end{array}$} & \multicolumn{2}{|c|}{ Área superficial $\left(\mathrm{mm}^{2} \mathrm{~cm}^{-3}\right)$} & \multicolumn{2}{|c|}{ Comprimento $\left(\mathrm{mm} \mathrm{cm}^{-3}\right)$} & \multicolumn{2}{|c|}{ Volume $\left(\mathrm{mm}^{3} \mathrm{~cm}^{-3}\right)$} \\
\hline & Irrigado & Não irrigado & Irrigado & Não irrigado & Irrigado & Não irrigado \\
\hline & \multicolumn{6}{|c|}{ Valores absolutos } \\
\hline $0-10$ & $625 \mathrm{~b}$ & $1.286 \mathrm{a}$ & $206 \mathrm{~b}$ & $439 \mathrm{a}$ & $179 \mathrm{~b}$ & $330 \mathrm{a}$ \\
\hline $10-20$ & $214 \mathrm{a}$ & $388 \mathrm{a}$ & $69 \mathrm{a}$ & $120 \mathrm{a}$ & $62 b$ & $124 \mathrm{a}$ \\
\hline $20-30$ & $280 \mathrm{a}$ & $397 \mathrm{a}$ & $86 a$ & $129 \mathrm{a}$ & $98 \mathrm{a}$ & $127 \mathrm{a}$ \\
\hline $30-40$ & $278 \mathrm{a}$ & $436 \mathrm{a}$ & $84 a$ & $133 a$ & $90 \mathrm{a}$ & $138 \mathrm{a}$ \\
\hline $40-50$ & $240 \mathrm{a}$ & $372 a$ & $76 a$ & $113 a$ & $71 \mathrm{a}$ & $116 \mathrm{a}$ \\
\hline \multirow[t]{2}{*}{ 50-60 } & $129 \mathrm{a}$ & $214 \mathrm{a}$ & $44 \mathrm{a}$ & $67 \mathrm{a}$ & $34 \mathrm{a}$ & $63 \mathrm{a}$ \\
\hline & \multicolumn{6}{|c|}{ Valores percentuais $(\%)$} \\
\hline $0-10$ & 35 & 42 & 36 & 44 & 34 & 37 \\
\hline $10-20$ & 12 & 13 & 12 & 12 & 12 & 14 \\
\hline $20-30$ & 16 & 13 & 15 & 13 & 18 & 14 \\
\hline $30-40$ & 16 & 14 & 15 & 13 & 17 & 15 \\
\hline $40-50$ & 14 & 12 & 13 & 11 & 13 & 13 \\
\hline $50-60$ & 7 & 7 & 8 & 7 & 6 & 7 \\
\hline CV geral (\%) & \multicolumn{2}{|c|}{49,1} & \multicolumn{2}{|c|}{44,2} & \multicolumn{2}{|c|}{45,4} \\
\hline
\end{tabular}

${ }^{(1)}$ Médias seguidas de letras iguais, minúsculas nas linhas, não diferem pelo teste de Tukey, a 5\% de probabilidade. 
perfil do solo em plantas de café arábica irrigadas, em comparação às não irrigadas. $\mathrm{O}$ diâmetro das raízes não diferiu entre as plantas irrigadas e não irrigadas, tendose observado raízes com maiores diâmetros, próximo ao tronco do cafeeiro e em menor profundidade do solo (Tabela 4 e Figura 4).

$\mathrm{O}$ crescimento radicular do cafeeiro tende a ser menor em condições em que o solo se mantém, na maior parte do tempo, na capacidade de campo e não em condições de estresse moderado, uma vez que, sob deficit hídrico, a planta produz mais ácido abcísico, o que reduz o efeito do etileno, inibidor do crescimento radicular em comprimento (Rena \& Guimarães, 2000). Além disso, a condição de alto teor de água no bulbo úmido interfere na aeração do solo, já que a água prontamente disponível dificulta a passagem do etileno pelos poros do solo e permite que se concentre em níveis suficientes para retardar o desenvolvimento radicular (Rena \& Guimarães, 2000; Barreto et al., 2006).

Nas plantas irrigadas, maiores área superficial, comprimento e volume de raízes não ultrapassaram $20 \mathrm{~cm}$ no sentido da entrelinha $\left(>800 \mathrm{~mm}^{2} \mathrm{~cm}^{-3}\right.$, $>280 \mathrm{~mm} \mathrm{~cm}^{-3} \mathrm{e}>240 \mathrm{~mm}^{3} \mathrm{~cm}^{-3}$, respectivamente), e, nas plantas não irrigadas, as raízes se concentraram até aproximadamente $70 \mathrm{~cm}$ de distância do tronco do cafeeiro (Figuras 1, 2 e 3). Em ambos os casos, a maior concentração de raízes ficou abaixo da projeção da copa do cafeeiro, que apresentava $1,25 \mathrm{~m}$ de raio limítrofe. De acordo com o manejo empregado na região, a adubação de cobertura é realizada a lanço e o fertilizante é distribuído sob a projeção da copa

Tabela 4. Desdobramento da interação significativa entre os tratamentos distâncias do tronco do cafeeiro e camadas de solo, quanto às variáveis área superficial, comprimento, volume e diâmetro de raízes, em plantas de cafeeiro conilon (Coffea canephora) $)^{(1)}$.

\begin{tabular}{|c|c|c|c|c|c|c|c|c|c|c|c|c|c|c|}
\hline \multirow{2}{*}{$\begin{array}{l}\text { Camada } \\
\text { de solo } \\
(\mathrm{cm})\end{array}$} & \multicolumn{10}{|c|}{$\begin{array}{l}\text { Distâncias no sentido da linha de plantio } \\
(\mathrm{cm})\end{array}$} & \multicolumn{3}{|c|}{$\begin{array}{l}\text { Distâncias no sentido da } \\
\text { entrelinha de plantio }(\mathrm{cm})\end{array}$} & \multirow{2}{*}{$\begin{array}{c}\mathrm{CV} \\
\text { geral } \\
(\%)\end{array}$} \\
\hline & 17 & 33 & 50 & 67 & 83 & 100 & 117 & 133 & 150 & 167 & 17 & 33 & 50 & \\
\hline \multicolumn{15}{|c|}{ Área superficial $\left(\mathrm{mm}^{2} \mathrm{~cm}^{-3}\right)$} \\
\hline $0-10$ & $1.606 \mathrm{ab}$ & $747 \mathrm{~cd}$ & $868 \mathrm{~cd}$ & $739 \mathrm{~cd}$ & $752 \mathrm{~cd}$ & $636 \mathrm{~d}$ & $468 d$ & $463 d$ & $516 \mathrm{~d}$ & $507 d$ & $1.294 b c$ & $2.115 \mathrm{a}$ & $1.709 \mathrm{ab}$ & \multirow{6}{*}{49,1} \\
\hline $10-20$ & $548 \mathrm{a}$ & $156 \mathrm{a}$ & $231 \mathrm{a}$ & $217 \mathrm{a}$ & $114 \mathrm{a}$ & $166 \mathrm{a}$ & $170 \mathrm{a}$ & $58 \mathrm{a}$ & $160 \mathrm{a}$ & $321 \mathrm{a}$ & $670 \mathrm{a}$ & $564 a$ & $539 a$ & \\
\hline $20-30$ & $818 \mathrm{a}$ & $243 \mathrm{abc}$ & $260 \mathrm{abc}$ & $318 \mathrm{abc}$ & $239 \mathrm{abc}$ & $144 b c$ & $97 \mathrm{c}$ & $64 c$ & $92 \mathrm{c}$ & $226 \mathrm{abc}$ & $562 \mathrm{abc}$ & $767 \mathrm{ab}$ & $567 \mathrm{abc}$ & \\
\hline $30-40$ & $747 \mathrm{ab}$ & 450abcd & $191 \mathrm{bcd}$ & $256 \mathrm{bcd}$ & $88 \mathrm{~cd}$ & $123 b c d$ & $212 \mathrm{bcd}$ & $128 \mathrm{bcd}$ & $98 \mathrm{~cd}$ & $61 d$ & $910 \mathrm{a}$ & $652 \mathrm{abcd}$ & $721 \mathrm{abc}$ & \\
\hline $40-50$ & $645 \mathrm{ab}$ & $516 a b c$ & $425 \mathrm{abc}$ & $303 a b c$ & $108 b c$ & $109 \mathrm{bc}$ & $122 b c$ & $136 b c$ & $0 \mathrm{c}$ & $117 \mathrm{bc}$ & $467 \mathrm{abc}$ & $787 \mathrm{a}$ & $243 a b c$ & \\
\hline$\underline{50-60}$ & $398 \mathrm{a}$ & $336 \mathrm{a}$ & $151 \mathrm{a}$ & $208 \mathrm{a}$ & $26 \mathrm{a}$ & $48 \mathrm{a}$ & $87 \mathrm{a}$ & $36^{\mathrm{a}}$ & $0 \mathrm{a}$ & $0 \mathrm{a}$ & $196 \mathrm{a}$ & $487 \mathrm{a}$ & $254 \mathrm{a}$ & \\
\hline \multicolumn{15}{|c|}{ Comprimento $\left(\mathrm{mm} \mathrm{cm}^{-3}\right)$} \\
\hline $0-10$ & $554 \mathrm{ab}$ & $270 \mathrm{~cd}$ & $302 \mathrm{~cd}$ & $253 \mathrm{~cd}$ & $267 \mathrm{~cd}$ & $214 d$ & $153 d$ & $144 d$ & $171 d$ & $173 d$ & $430 \mathrm{bc}$ & $680 \mathrm{a}$ & $576 \mathrm{ab}$ & \multirow{6}{*}{44,2} \\
\hline $10-20$ & $181 \mathrm{a}$ & $53 a$ & $79 a$ & $57 \mathrm{a}$ & $36 a$ & $56 \mathrm{a}$ & $53 a$ & $21^{\mathrm{a}}$ & $53 a$ & $106 a$ & $189 \mathrm{a}$ & $179 a$ & $165 \mathrm{a}$ & \\
\hline $20-30$ & $248 \mathrm{a}$ & $67 \mathrm{ab}$ & $109 \mathrm{ab}$ & $96 \mathrm{ab}$ & $73 \mathrm{ab}$ & $50 \mathrm{ab}$ & $88 \mathrm{ab}$ & $22 b$ & $15 \mathrm{~b}$ & $75 \mathrm{ab}$ & $176 \mathrm{ab}$ & $217 \mathrm{ab}$ & $163 \mathrm{ab}$ & \\
\hline $30-40$ & $234 a b$ & $142 \mathrm{abc}$ & $60 \mathrm{abc}$ & $86 a b c$ & $28 b c$ & $44 a b c$ & $74 a b c$ & $39 \mathrm{bc}$ & $35 \mathrm{bc}$ & $21 \mathrm{c}$ & $254 a$ & $203 \mathrm{abc}$ & $186 \mathrm{abc}$ & \\
\hline $40-50$ & $200 \mathrm{ab}$ & $155 \mathrm{ab}$ & $133 a b$ & $99 \mathrm{ab}$ & $31 \mathrm{ab}$ & $36 \mathrm{ab}$ & $40 \mathrm{ab}$ & $44 \mathrm{ab}$ & $0 b$ & $35 \mathrm{ab}$ & $144 \mathrm{ab}$ & $231 \mathrm{a}$ & $80 \mathrm{ab}$ & \\
\hline $50-60$ & $144 \mathrm{a}$ & $112 \mathrm{a}$ & $45 \mathrm{a}$ & $69 a$ & $9 \mathrm{a}$ & $12 \mathrm{a}$ & $27 \mathrm{a}$ & $13 \mathrm{a}$ & $0 \mathrm{a}$ & $0 \mathrm{a}$ & $66 a$ & $152 \mathrm{a}$ & $75 a$ & \\
\hline \multicolumn{15}{|c|}{ Volume $\left(\mathrm{mm}^{3} \mathrm{~cm}^{-3}\right)$} \\
\hline $0-10$ & $406 \mathrm{abc}$ & $182 \mathrm{de}$ & 218 cde & $187 \mathrm{de}$ & $181 \mathrm{de}$ & $169 \mathrm{de}$ & $137 \mathrm{e}$ & $151 \mathrm{de}$ & $138 \mathrm{e}$ & $134 \mathrm{e}$ & $349 \mathrm{bcd}$ & $594 a$ & $461 \mathrm{ab}$ & \multirow{6}{*}{45,4} \\
\hline $10-20$ & $159 \mathrm{ab}$ & $40 \mathrm{~b}$ & $60 \mathrm{ab}$ & $94 \mathrm{ab}$ & $34 b$ & $43 b$ & $50 \mathrm{~b}$ & $14 b$ & $43 b$ & $86 \mathrm{ab}$ & $251 \mathrm{a}$ & $167 \mathrm{ab}$ & $171 \mathrm{ab}$ & \\
\hline $20-30$ & $272 \mathrm{ab}$ & $87 \mathrm{abcd}$ & $67 \mathrm{~cd}$ & 99abcd & $73 \mathrm{bcd}$ & $36 \mathrm{~cd}$ & $30 \mathrm{~cd}$ & $16 \mathrm{~d}$ & $31 \mathrm{~cd}$ & $63 \mathrm{~cd}$ & $220 \mathrm{abc}$ & 280abcd & 189abcd & \\
\hline $30-40$ & $228 \mathrm{abc}$ & $123 \mathrm{bcd}$ & $55 \mathrm{~cd}$ & $67 \mathrm{~cd}$ & $24 d$ & $30 \mathrm{~cd}$ & $52 \mathrm{~cd}$ & $42 \mathrm{~cd}$ & $24 d$ & $16 \mathrm{~d}$ & $325 a$ & $204 \mathrm{abcd}$ & $294 \mathrm{ab}$ & \\
\hline $40-50$ & $194 \mathrm{ab}$ & $159 \mathrm{ab}$ & $124 \mathrm{ab}$ & $82 \mathrm{ab}$ & $37 b$ & $30 \mathrm{~cd}$ & $34 b$ & $38 b$ & $1 b$ & $37 b$ & $140 \mathrm{ab}$ & $272 \mathrm{a}$ & $67 b$ & \\
\hline$\underline{50-60}$ & $94 \mathrm{a}$ & $89 a$ & $51 \mathrm{a}$ & $55 \mathrm{a}$ & $8 \mathrm{a}$ & $18 \mathrm{a}$ & $25 \mathrm{a}$ & $10 \mathrm{a}$ & $1 \mathrm{a}$ & $1 \mathrm{a}$ & $51 \mathrm{a}$ & $146 \mathrm{a}$ & $79 \mathrm{a}$ & \\
\hline \multicolumn{15}{|c|}{ Diâmetro (mm) } \\
\hline $0-10$ & $0,75 \mathrm{a}$ & $0,72 \mathrm{a}$ & $0,75 \mathrm{a}$ & $0,78 \mathrm{a}$ & $0,76 \mathrm{a}$ & $0,77 \mathrm{a}$ & $0,75 \mathrm{a}$ & $0,77 \mathrm{a}$ & $0,78 \mathrm{a}$ & $0,68 \mathrm{a}$ & $0,79 a$ & $0,80 \mathrm{a}$ & $0,71 \mathrm{a}$ & \multirow{6}{*}{1,51} \\
\hline $10-20$ & $0,74 \mathrm{a}$ & $0,59 \mathrm{a}$ & $0,59 \mathrm{a}$ & $0,67 \mathrm{a}$ & $0,45 \mathrm{a}$ & $0,44 \mathrm{a}$ & $0,52 \mathrm{a}$ & $0,38 \mathrm{a}$ & $0,36 \mathrm{a}$ & $0,61 \mathrm{a}$ & $0,77 \mathrm{a}$ & $0,78 \mathrm{a}$ & $0,77 \mathrm{a}$ & \\
\hline $20-30$ & $0,77 \mathrm{a}$ & $0,54 \mathrm{abcd}$ & $0,76 \mathrm{a}$ & $0,68 \mathrm{ab}$ & $0,46 a b c d$ & $0,29 \mathrm{bcd}$ & 0,39abcd & $0,16 \mathrm{~d}$ & $0,23 \mathrm{~cd}$ & $0,69 \mathrm{ab}$ & $0,68 \mathrm{ab}$ & $0,71 \mathrm{ab}$ & $0,62 \mathrm{ab}$ & \\
\hline $30-40$ & $0,76 \mathrm{a}$ & $0,78 \mathrm{a}$ & $0,45 \mathrm{abc}$ & $0,44 \mathrm{abc}$ & $0,38 \mathrm{abc}$ & $0,44 \mathrm{abc}$ & $0,45 \mathrm{abc}$ & $0,23 \mathrm{c}$ & $0,29 b c$ & $0,23 \mathrm{c}$ & $0,70 \mathrm{ab}$ & $0,71 \mathrm{ab}$ & $0,77 \mathrm{a}$ & \\
\hline $40-50$ & $0,77 \mathrm{a}$ & $0,70 \mathrm{ab}$ & $0,53 \mathrm{ab}$ & $0,61 \mathrm{ab}$ & $0,39 \mathrm{abc}$ & $0,31 b c$ & $0,30 \mathrm{bc}$ & $0,30 \mathrm{bc}$ & $0,00 \mathrm{c}$ & $0,30 \mathrm{bc}$ & $0,71 \mathrm{ab}$ & $0,71 \mathrm{ab}$ & $0,52 \mathrm{ab}$ & \\
\hline $50-60$ & $0,66 \mathrm{a}$ & $0,54 \mathrm{abc}$ & 0,43 abcd & 0,39 abcde & $0,13 \mathrm{cde}$ & $0,07 \mathrm{de}$ & 0,22 bcde & $0,08 \mathrm{de}$ & $0,00 \mathrm{e}$ & $0,00 \mathrm{e}$ & $0,47 \mathrm{abcd}$ & $0,61 \mathrm{ab}$ & 0,37 abcde & \\
\hline
\end{tabular}

${ }^{(1)}$ Médias seguidas de letras iguais, minúsculas nas linhas, não diferem pelo teste de Tukey, a 5\% de probabilidade. 
do cafeeiro, a, aproximadamente, 1,0 $\mathrm{m}$ do tronco. Contudo, os resultados obtidos no presente trabalho são indicativos de que as aplicações de fertilizantes em cobertura poderiam ser realizadas na região compreendida pela linha de plantio do cafeeiro, que apresenta maior concentração de raízes, isto é, entre 10 e $50 \mathrm{~cm}$ do tronco do cafeeiro, distância que pode chegar a $70 \mathrm{~cm}$ em lavouras não irrigadas.

$\mathrm{Na}$ camada de $0-10 \mathrm{~cm}$, observaram-se 35,36 e $34 \%$ de área superficial, comprimento e volume de raízes, respectivamente, nas plantas irrigadas (Tabela 3). Nessa mesma camada, verificaram-se 42, 44 e $37 \%$ de área superficial, comprimento e volume de raízes, respectivamente, nas plantas não irrigadas (Tabela 3). Já na camada de 50 a $60 \mathrm{~cm}$ de profundidade, a percentagem de raízes encontradas não passou de $8 \%$, para as características avaliadas. Foram constatados valores percentuais próximos, entre 11 e $18 \%$, para área superficial, comprimento e volume de raízes, nas camadas de 10-20, 2030, 30-40 e 40-50 cm (Tabela 3). Partelli et al. (2014) observaram percentual superior a $55 \%$ de raízes na camada de 0 a $20 \mathrm{~cm}$ do solo, ao avaliar área superficial, comprimento e volume de raízes. Resultados semelhantes foram relatados por Motta et al. (2006), que encontraram maior comprimento ( $\mathrm{cm} \mathrm{dm}{ }^{-3}$ de solo) de raízes de cafeeiro arábica em até $20 \mathrm{~cm}$ de profundidade, e por Rodrigues et al. (2001), que verificaram menor área superficial e comprimento de raízes com o aumento da profundidade em cafeeiros cultivados sob diferentes concentrações de alumínio. O maior volume do sistema radicular nas camadas superiores do solo pode ser explicado pela ausência de uma raiz pivotante proeminente e pelas adubações realizadas na superfície do solo (Rena \& Guimarães, 2000).
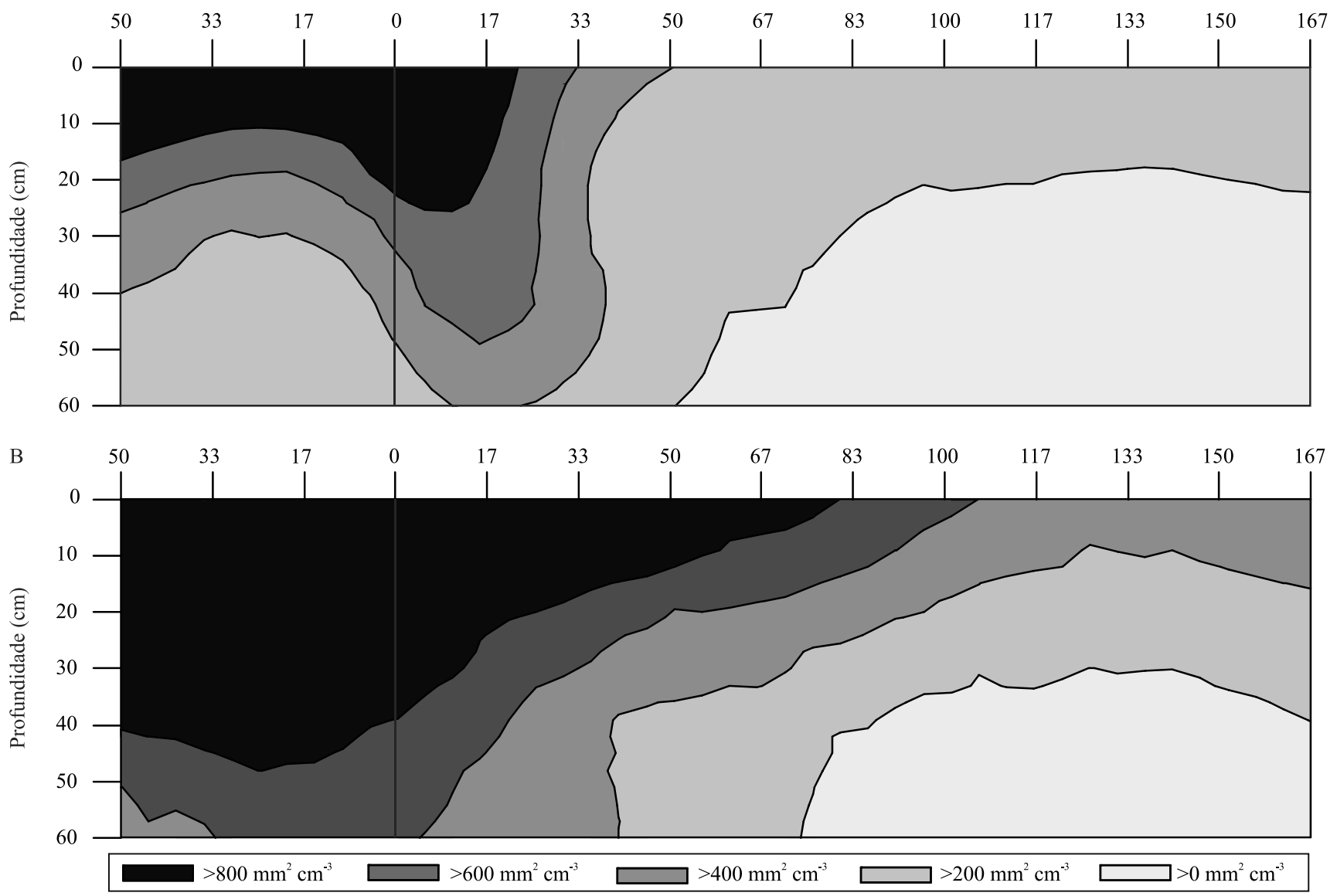

Figura 1. Distribuição espacial da área superficial das raízes de cafeeiro conilon (Coffea canephora) irrigado (A) e não irrigado (B), em diferentes distâncias e profundidades. O ponto zero refere-se à localização da planta. 
Menores área superficial, comprimento e volume de raízes foram observados a partir de $50 \mathrm{~cm}$ de profundidade, o que coincidiu com: a menor concentração de nutrientes (fósforo, cálcio, magnésio, zinco, cobre e manganês) e matéria orgânica; a presença de alumínio; o aumento da acidez potencial do solo $(\mathrm{H}+\mathrm{Al})$; a redução do $\mathrm{pH}$; e o aumento do teor de argila (Tabela 1), que pode estar associado ao aumento da densidade do solo. Partelli et al. (2014) e Ronchi et al. (2015), ao avaliar o sistema radicular de plantas de C. canephora adultas e de cafeeiro arábica em franco crescimento, respectivamente, também constataram que, na área superficial, o comprimento e o volume de raízes reduziu com a profundidade no perfil do solo, assim como o diâmetro médio de raízes, com o empobrecimento da fertilidade do solo. Embora o sistema radicular do cafeeiro apresente características de desenvolvimento ligadas, principalmente, à genética da planta, outros fatores podem modificar sua distribuição espacial, como tipo de propagação, formação das mudas e, sobretudo, quantidade de água no solo (Franco \& Inforzato, 1946; Partelli et al., 2014). A baixa fertilidade nas camadas mais profundas do solo pode ser um fator limitante para o desenvolvimento do sistema radicular do cafeeiro nos solos brasileiros (Rena \& Guimarães, 2000), em especial nos tabuleiros costeiros.

Ronchi et al. (2015) observaram, em lavoura jovem (27 a 39 meses após o transplantio) irrigada, que o sistema radicular nas camadas mais profundas $(40 \mathrm{~cm})$ estava em pleno crescimento. Isso também foi verificado no presente trabalho, uma vez que a lavoura ainda não tinha sido submetida à poda de ramos ortotrópicos.

A Distância no sentido da linha (cm)
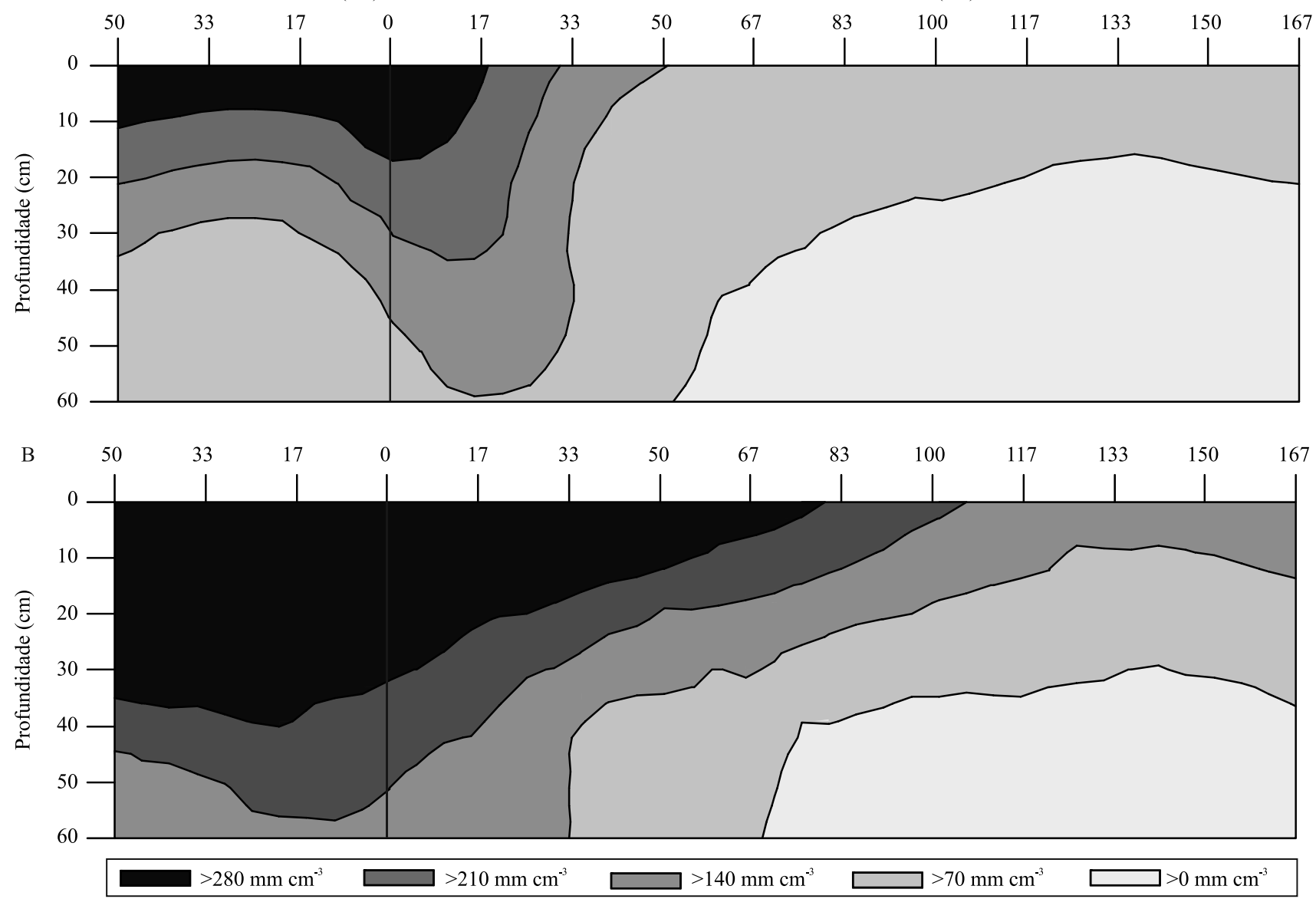

Figura 2. Distribuição espacial do comprimento das raízes de cafeeiro conilon (Coffea canephora) irrigado (A) e não irrigado (B), em diferentes distâncias e profundidades. O ponto zero refere-se à localização da planta. 


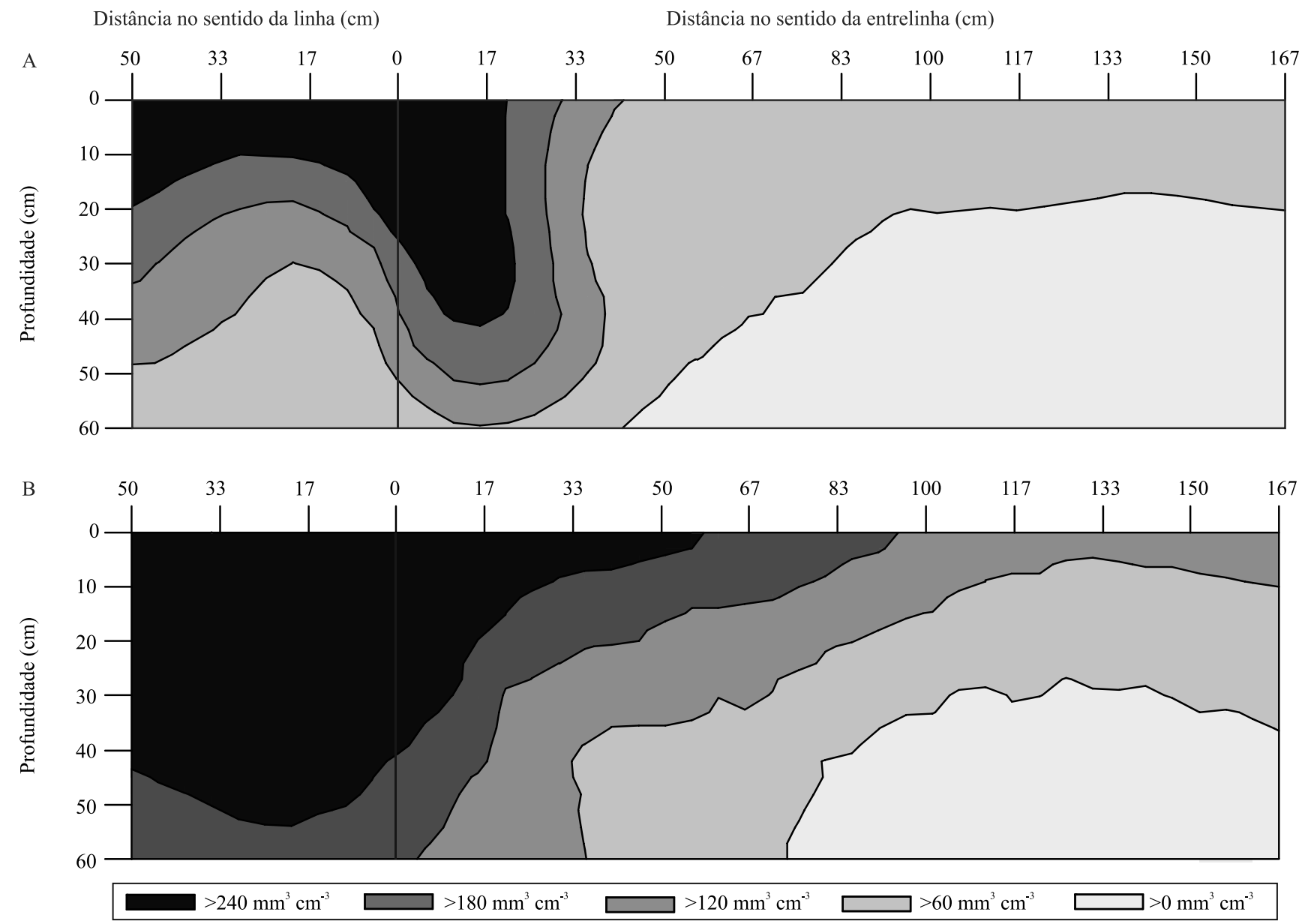

Figura 3. Distribuição espacial do volume das raízes de cafeeiro conilon (Coffea canephora) irrigado (A) e não irrigado (B), em diferentes distâncias e profundidades. O ponto zero refere-se à localização da planta.

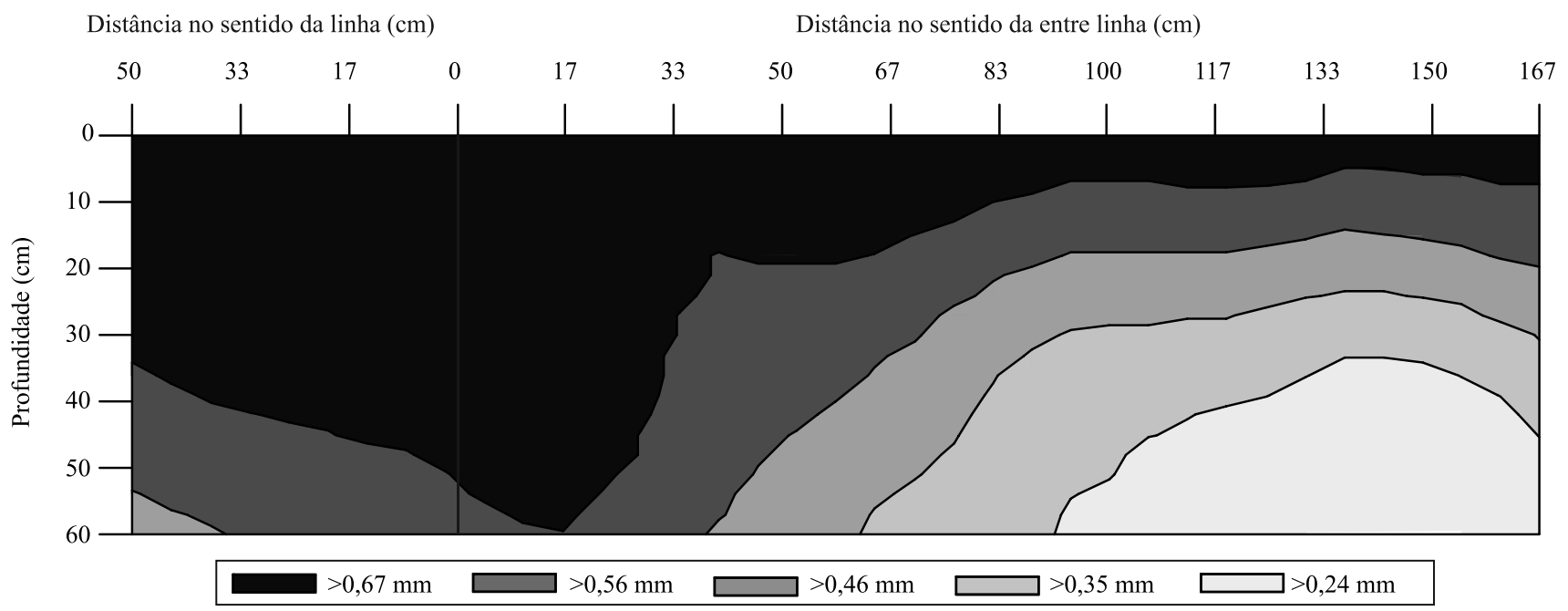

Figura 4. Distribuição espacial do diâmetro das raízes de cafeeiro conilon (Coffea canephora) irrigado e não irrigado, em diferentes distâncias e profundidades. O ponto zero refere-se à localização da planta. 


\section{Conclusões}

1. As plantas de café conilon (Coffea canephora) não irrigadas apresentam maiores área superficial, comprimento e volume de raízes por volume de solo na camada superior, bem como distribuição menos discrepante de raízes na superfície do solo e em profundidade, em comparação às plantas irrigadas.

2. O sistema de irrigação por gotejamento promove maior quantidade de raízes nas plantas irrigadas, na zona compreendida pelo bulbo úmido da irrigação.

\section{Agradecimentos}

Ao Conselho Nacional de Desenvolvimento Científico e Tecnológico (CNPq), pelo apoio financeiro e aos produtores Ademir Trevizani, Daniel Trevizani e Luiz Antônio Covre, pelo apoio na consecução do trabalho.

\section{Referências}

ARAUJO, G.L.; REIS, E.F. dos; MORAES, W.B.; GARCIA, G. de O.; NAZÁRIO, A.A. Influência do déficit hídrico no desenvolvimento inicial de duas cultivares de café conilon. Irriga, v.16, p.115-124, 2011. DOI: 10.15809/irriga.2011v16n2p115.

BARRETO, C.V.G.; SAKAI, E.; ARRUDA, F.B.; SILVA, E.A. da; PIRES, R.C. de M. Distribuição espacial do sistema radicular do cafeeiro fertirrigado por gotejamento em Campinas. Bragantia, v.65, p.641-647, 2006. DOI: 10.1590/S0006-87052006000400015.

BELAN, L.L.; SILVA, K.G.; TOMAZ, M.A.; JESUS JUNIOR, W.C.; AMARAL, J.A.T. do; AMARAL, J.F.T. do. Aspectos fisiológicos do cafeeiro conilon: uma abordagem sistemática. Nucleus, v.8, p.225-240, 2011. DOI: 10.3738/1982.2278.551.

BRAGANÇA, S.M.; CARVALHO, C.H.S. de; FONSECA, A.F.A. da; FERRÃO, R.G. Variedades clonais de café conilon para o Estado do Espírito Santo. Pesquisa Agropecuária Brasileira, v.36, p.765-770, 2001. DOI: 10.1590/S0100-204X2001000500006.

CARVALHO, M.; JESUS, A.M.S.; CARVALHO, S.P. de; GOMES, C.N.; SOARES, A.M. Comportamento em condições de campo de cafeeiros (Coffea arabica L.) propagados vegetativamente e por semeadura. Coffee Science, v.3, p.108-114, 2008.

DAMATTA, F.M.; RAMALHO, J.D.C. Impacts of drought and temperature stress on coffee physiology and production: a review. Brazilian Journal of Plant Physiology, v.18, p.55-81, 2006. DOI: 10.1590/S1677-04202006000100006.

FRANCO, C.M.; INFORZATO, R. O sistema radicular do cafeeiro nos principais tipos de solo do estado de São Paulo. Bragantia, v.6, p.443-478, 1946. DOI: 10.1590/S0006-87051946000900001.

HOOGENBOOM, G.; HUCK, M.G.; PETERSON, C.M. Root growth rate of soybean as affected by drought stress. Agronomy
Journal, v.79, p.607-614, 1987. DOI: 10.2134/agronj1987.00021 $962007900040004 x$.

LYNCH, J. Root architecture and plant productivity. Plant Physiology, v.109, p.7-13, 1995. DOI: 10.1104/pp.109.1.7.

MARRACCINI, P.; VINECKY, F.; ALVES, G.S.C.; RAMOS, H.J.O.; ELBELT, S.; VIEIRA, N.G.; CARNEIRO, F.A.; SUJII, P.S.; ALEKCEVETCH, J.C.; SILVA, V.A.; DAMATTA, F.M.; FERRÃO, M.A.G.; LEROY, T.; POT, D.; VIEIRA, L.G.E.; SILVA, F.R. da; ANDRADE, A.C. Differentially expressed genes and proteins upon drought acclimation in tolerant and sensitive genotypes of Coffea canephora. Journal of Experimental Botany, v.63, p.4191-4212, 2012. DOI: 10.1093/jxb/ers103.

MOTTA, A.C.V.; NICK, J.A.; YORINORI, G.T.; SERRAT, B.M. Distribuição horizontal e vertical da fertilidade do solo e das raízes de cafeeiro (Coffea arabica L.) cultivar Catuaí. Acta Scientiarum. Agronomy, v.28, p.455-463, 2006. DOI: 10.4025/actasciagron. v28i4.758.

PARTELLI, F.L.; COVRE, A.M.; OLIVEIRA, M.G.; ALEXANDRE, R.S.; VITÓRIA, E.L. da; SILVA, M.B. da. Root system distribution and yield of 'Conilon' coffee propagated by seeds or cuttings. Pesquisa Agropecuária Brasileira, v.49, p.349-355, 2014. DOI: 10.1590/S0100-204X2014000500004.

PARTELLI, F.L.; MARRÉ, W.B.; FALQUETO, A.R.; VIEIRA, H.D.; CAVATTI, P.C. Seasonal vegetative growth in genotypes of Coffea canephora, as related to climatic factors. Journal of Agricultural Science, v.5, p.108-116, 2013. DOI: 10.5539/jas. v5n8p108.

PINHEIRO, H.A.; DAMATTA, F.M.; CHAVES, A.R.M.; FONTES, E.P.B.; LOUREIRO, M.E. Drought tolerance in relation to protection against oxidative stress in clones of Coffea canephora subjected to long-term drought. Plant Science, v.167, p.1307-1314, 2004. DOI: 10.1016/j.plantsci.2004.06.027.

RENA,A.B.; GUIMARÃES, P.T.G. Sistema radicular do cafeeiro: estrutura, distribuição, atividade e fatores que o influenciam. Belo Horizonte: Epamig, 2000. 80p. (Epamig. Documentos, 37).

RODRIGUES, L.A.; MARTINEZ, H.E.P.; NEVES, J.C.L.; NOVAIS, R.F.; MENDONÇA, S.M. Growth response of coffee tree shoots and roots to subsurface liming. Plant and Soil, v.234, p.207-214, 2001. DOI: 10.1023/A:1017999318532.

RONCHI, C.P.; SOUSA JÚNIOR, J.M. de; AMEIDA, W.L. de; SOUZA, D.S.; SILVA, N.O.; OLIVEIRA, L.B. de; GUERRA, A.M.N. de M.; FERREIRA, P.A. Morfologia radicular de cultivares de café arábica submetidas a diferentes arranjos espaciais. Pesquisa Agropecuária Brasileira, v.50, p.187-195, 2015. DOI: 10.1590/ S0100-204X2015000300001.

SAKAI, E.; BARBOSA, E.A.A.; SILVEIRA, J.M. de C.; PIRES, R.C. de M. Coffee productivity and root systems in cultivation schemes with different population arrangements and with and without drip irrigation. Agricultural Water Management, v.148, p.16-23, 2015. DOI: 10.1016/j.agwat.2014.08.020.

SANTOS, H.G. dos; JACOMINE, P.K.T.; ANJOS, L.H.C. dos; OLIVEIRA, V.A. de; LUMBRERAS, J.F.; COELHO, M.R.; ALMEIDA, J.A. de; CUNHA, T.J.F.; OLIVEIRA, J.B. de. Sistema brasileiro de classificação de solos. 3.ed. Brasília: Embrapa, 2013. 353p.

Pesq. agropec. bras., Brasília, v.50, n.11, p.1006-1016, nov. 2015 DOI: 10.1590/S0100-204X2015001100003 
SANTOS, R.F.; CARLESSO, R. Déficit hídrico e os processos morfológico e fisiológico das plantas. Revista Brasileira de Engenharia Agrícola e Ambiental, v.2, p.287-294, 1998.

SILVA, A.L. da; BRUNO, I.P.; REICHARDT, K.; BACCHI, O.O.S.; DOURADO-NETO, D.; FAVARIN, J.L.; COSTA, F.M.P. da; TIMM, L.C. Soil water extraction by roots and Kc for the coffee crop. Revista Brasileira de Engenharia Agrícola e Ambiental, v.13, p.257-261, 2009. DOI: 10.1590/S1415-43662009000300006.

SILVA, P.E.M.; CAVATTE, P.C.; MORAIS, L.E.; MEDINA, E.F.; DAMATTA, F.M. The functional divergence of biomass partitioning, carbon gain and water use in Coffea canephora in response to the water supply: implications for breeding aimed at improving drought tolerance. Environmental and
Experimental Botany, v.87, p.49-57, 2013. DOI: 10.1016/j. envexpbot.2012.09.005.

SILVA, V.A.; ANTUNES, W.C.; GUIMARÃES, B.L.S.; PAIVA, R.M.C.; SILVA, V. de F.; FERRÃO, M.A.G.; DAMATTA, F.M.; LOUREIRO, M.E. Resposta fisiológica de clone de café Conilon sensível à deficiência hídrica enxertado em porta-enxerto tolerante. Pesquisa Agropecuária Brasileira, v.45, p.457-464, 2010. DOI: 10.1590/S0100-204X2010000500004.

VERDIN FILHO, A.C.; TOMAZ, M.A.; FERRÃO, R.G.; FERRÃO, M.A.G.; FONSECA, A.F.A. da; RODRIGUES, W.N. Conilon coffee yield using the programmed pruning cycle and different cultivation densities. Coffee Science, v.9, p.489-494, 2014.

Recebido em 30 de junho de 2015 e aprovado em 16 de setembro de 2015 